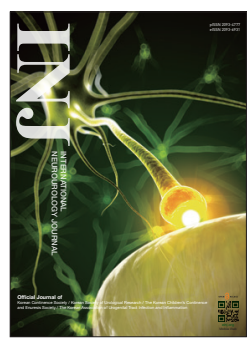

\title{
Neuropelveology: An Emerging Discipline for the Management of Chronic Pelvic Pain
}

\author{
Marc Possover, Karl-Erik Andersson, Axel Forman \\ Department of Obstetrics and Gynaecology, Aarhus University Hospital, Aarhus, Denmark
}

\begin{abstract}
Chronic pelvic pain (CPP) is a common condition involving multiple, organ-specific medical specialties, each with its own approach to diagnosis and treatment. Management requires knowledge of the interplay between pelvic organ function and neuro-functional anatomy, and of the neurologic and psychological aspects of CPP, but no current specialty fully encompasses this approach. Neuropelveology is an emerging discipline focusing on pathologies of the pelvic nervous system on a cross-disciplinary basis. It involves a neurological/neurosurgical approach, combining the knowledge required for a proper neurologic diagnosis, confirmation by transvaginal/transrectal examination of the pelvic nerves, and advanced laparoscopic surgery in selected cases of CPP. The management of CPP requires multidisciplinary contributions, and neuropelveology may offer an educational framework for the interdisciplinary exchange of knowledge between clinical physicians and basic researchers.
\end{abstract}

Keywords: Neuropelveology; Pelvic Pain; Nerve; Plexus

- Conflict of Interest: No potential conflict of interest relevant to this article was reported.

\section{INTRODUCTION}

The pelvis is a meeting point for multiple specialties, such as urology, gynaecology, medical and surgical gastroenterology, orthopaedic surgery, and physiotherapy. Chronic pelvic pain (CPP), often with urogenital, bowel, or pelvic floor dysfunction, is a frequent problem across these disciplines. In light of the close relations between the pelvic organs and the visceral and somatic nerve plexuses, it is not surprising that most cases of pelvic pathology have such symptoms in common. Making a specific diagnosis in cases of pelvic pain is therefore difficult, and requires a detailed understanding of the neurology and neuropathology specific to the area, but these specialties are rarely involved. The clinical process is therefore hampered by deficient knowledge, as well as insufficient methods for visualisation and intervention.
We hereby suggest the introduction of neuropelveology as a new discipline with a focus on these problems.

\section{CHRONIC PELVIC PAIN}

CPP is defined as nonmalignant pain perceived in pelvic structures [1]. In some patients, this occurs as a consequence of welldefined pathology, such as infection, endometriosis, haemorrhoids, anal fissure, pudendal neuropathy, sacral spinal cord pathology, vascular and cutaneous diseases, or psychiatric conditions. When pain continues for more than 6 months after the elimination of the initial pathology or when no cause is found, chronic pelvic pain syndrome (CPPS) is considered to have developed [1]. Estimates of CPPS prevalence range from $4 \%$ to $15 \%[2,3]$. The syndrome is often accompanied by symptoms

Corresponding author: Axel Forman (iD https://orcid.org/0000-0003-1369-1296 Department of Obstetrics and Gynaecology, Aarhus University Hospital, Palle Juul-Jensens Boulevard 99, 8200 Aarhus C, Denmark

E-mail: af@clin.au.dk / Tel: +45-78453354 / Fax: +45-78453393

Submitted: November 18, 2017 / Accepted after revision: December 6, 2017 
related to organs in the pelvis, but without any obvious pathology $[1,4]$. Negative cognitive, behavioural, sexual, or emotional consequences and impaired quality of life are frequent problems for patients with CPPS [1], and many have to accept longterm medical pain treatment [5].

Pathology of the visceral and somatic pelvic neuronal plexuses might explain a significant part of unspecified pelvic pain and associated pelvic organ dysfunctions, making a detailed understanding of their properties necessary. Visceral pain is conveyed through the hypogastric plexus, where changes in nerve composition and pain sensation are seen [6]. Thus, afferent autonomic signalling may induce diffuse reflex mechanisms with a decreased pain threshold in organs distant from the pathologic lesion. This has been demonstrated in a rat model in which mesenteric endometriosis produced diffuse autonomous reflex dysfunction with hyperalgesia in abdominal organs distant from the lesion $[7,8]$, as well as bladder inflammation with decreased capacity [9]. In line with these experimental findings, endometriosis patients may suffer from hyperalgesia in the lower abdominal organs innervated by the autonomic nervous system. The clinical consequences may include deep dyspareunia, bladder pain syndrome, and irritable bowel syndrome [10]; moreover, a visceral syndrome, which was defined as including multiple abdominal pain symptoms, was seen more often in endometriosis patients than in women without the disease [11]. These findings call for a better understanding of autonomic neuronal function in CPPS in order to develop new therapeutic strategies. Of interest, sacral nerve stimulation has been proposed for treatment of painful bladder syndrome [12], and the further development of such strategies is warranted.

Neurosurgical techniques are well established for nerve lesions of the upper limb, but surgical exploration of the pelvic retroperitoneal area and the pelvic nerves is still uncommon. The only documented pelvic nerve pathology is pudendal neuralgia (Alcock canal syndrome) [13], probably because the nerve is easily accessible for neurophysiological exploration, infiltration, and surgical decompression. In contrast, the endopelvic somatic nerves, especially the sacral plexus, are difficult to access and have been less thoroughly investigated in the past, although pathologies of these structures might explain some cases of CPP/CPPS [14].

\section{NEUROPELVEOLOGY}

Neuropelveology has been introduced as a new discipline fo- cused on pathologies of the pelvic nervous system and the possibilities for improved neurologic diagnosis in CPP/CPPS [15]. Because of growing interest from the medical community, the International Society of Neuropelveology (ISoN, www.theison. org) was founded in 2014 to provide universal access to education in this area.

Standard medical training imparts the concept that the location of the pain and its aetiology correspond to the same area. The classical workup usually follows 2 steps: determination of the location of the pain, followed by determination of a potential aetiology in the corresponding anatomical area. This second step is based on a clinical examination and sonography and/or magnetic resonance imaging (MRI), typically followed by laparoscopic exploration of the pelvis for confirmation and treatment of the suspected aetiology.

The neuropelveological approach to pelvic neuropathies is primarily diagnostic, involving the application of neurological principles and a comprehensive knowledge of pelvic neurofunctional anatomy. Patient's history is the key, with a focus not only on the location of the pain, but also on the history of the pain, its radiation, aggravating factors, and vegetative and somatic symptoms. Taking the patient's history is time-consuming, but in most cases it enables the establishment of a potential diagnosis, which can be substantiated or rejected by the clinical examination. The first step is to evaluate whether the pain is visceral or somatic.

Visceral low abdominal pain is mediated through the hypogastric plexus and can be recognized due to its diffuse character, sometimes with radiation to the back and multiple vegetative symptoms such as malaise, oppression, syncope, irritability, bloating, nausea, vomiting, and fatigue. The clinical examination should include signs of autonomous dysfunction such as tachycardia, pupil dilatation, and tachycardia with hypotension [16]. Making a specific clinical diagnosis of the site and extent of the underlying pathology is difficult, except when local lesions, such as deep infiltrating endometriosis, can be palpated or diagnosed on MRI. Diagnostic laparoscopy performed in referral centres for treatment of the underlying pathology may be warranted, and a knowledge of the autonomous neuronal dysfunction underlying the multiple symptoms is important in the approach to the patient.

In somatic pain, it is essential to adopt a 'classic neurological way of thinking, since the location of the pain and the site of the potential corresponding lesions can differ. Somatic pain is located superficially at the skin and is described as allodynia or 
electrical shock, with a specific location, caudal radiation to the genito-anal areas or to the lower extremities, and a lack of vegetative symptoms. The neuropelveological workup of suspected somatic pelvic pain follows 6 steps:

(1) Determination of the nerve pathways involved in relaying pain information to the brain. Inguino-abdominal pain with radiation to the ventral lower abdomen and genital area is correlated with pathology of branches from the lumbar plexus. Genito-anal/pudendal, coccygeal, and pelvic pain with caudal radiation in the sacral dermatomes is correlated with pathology of the sacral plexus or its branches, such as the sciatic gluteal or pudendal nerves.

(2) Determination of the location of neurological irritation/ injury (truncular vs. radicular vs. central nervous system).

(3) Determination of the type of nerve lesion (irritation vs. injury [axonal lesion]) based on a classic neurological examination of the lumbosacral nerves and urodynamic testing. Sensorimotor dysfunction of the sacral nerves, which manifests as bladder hypotonia or atonia, erectile dysfunction, and constipation, is correlated with axonal lesions of the nerves. Hyperesthesia and bladder hypersensitivity/overactivity or even persistent genital arousal disorder may be correlated with irritation of these nerves $[17,18]$.

(4) Neurological confirmation of the suspected diagnosis by (a) examination of the distal branches of the lumbar plexus (e.g., the cremaster reflex in males), (b) transvaginal or transrectal palpation of the sacral plexus and of the pudendal nerve with reproduction of the trigger pain and the Tinel sign when present, in line with classic neurologic examination techniques, and (c) selective anaesthetic blockade of the nerve(s).

(5) Determination of a potential aetiology based on the patient's history and the clinical findings (e.g., previous surgery, pelvic congestion syndrome, May-Turner syndrome, pelvic trauma, obstetric procedures), followed by transvaginal or transrectal Doppler sonography at the trigger point and/or neuro-MRI if available.

(6) Final definition of the suspected aetiology and proposal for the corresponding treatment. In many of these cases, an aetiologic diagnosis may be reached, and proper treatment instituted. However, this is a field where research into new strategies is needed.

Because somatic neuropathic pain is more specific, the neu- ropelveological workup typically enables diagnosis of the lesion site in the pelvic nerves [18]. Intervention in this area, which is covered by large vessels and a dense network of lymph nodes, has hitherto been hindered by the lack of minimally invasive surgical methods. However, developments in video endoscopy have enabled the exploration of the retroperitoneal pelvic space with access to the lumbosacral plexus. This has enabled the diagnosis of hitherto unknown local causes of somatic pelvic pain, such as vascular entrapment or local fibrosis secondary to previous surgery, as well as nerve decompression or neurolysis performed in a 1-step procedure [18-21]. In cases of axonal lesions, this endoscopic approach further permits the laparoscopic implantation of neuroprosthesis (LION) procedure, in which electrodes are selectively placed in contact with the pelvic nerves $[22,23]$. This may allow pain to be controlled, in parallel to neuromodulation in other peripheral nerves [24], and this approach could also represent an alternative to current methods for sacral root stimulation $[25,26]$. Moreover, recent studies indicate that the LION procedure applied to the sciatic and femoral nerves might engage residual spinal and peripheral pathways for the recovery of voluntary motion of the legs in patients with chronic paraplegia secondary to spinal cord injury $[27,28]$. These neuropelveological procedures should be reserved for experienced surgeons with special training in laparoscopic retroperitoneal pelvic surgery, but the diagnosis of pelvic nerve pathologies is possible for all clinicians familiar with the neurological symptoms and signs specific to the area $[29,30]$.

\section{CONCLUSION}

It seems reasonable to advise colleagues dealing with CPPS patients to apply neuropelveological principles in their clinical work. The ISoN offers an e-learning program for acquisition of the necessary knowledge, and this program is accessible to all physicians at the ISoN homepage (www.theison.org). This evolution in the management of patients suffering from CPPS requires more communication between neurology, the pelvic clinical disciplines, and basic research. Promotion of this process represents the aim of the new specialty of neuropelveology.

\section{REFERENCES}

1. Engeler DS, Baranowski AP, Dinis-Oliveira P, Elneil S, Hughes J, Messelink EJ, et al. The 2013 EAU guidelines on chronic pelvic pain: is management of chronic pelvic pain a habit, a philosophy, 
or a science? 10 years of development. Eur Urol 2013;64:431-9.

2. Howard FM. Chronic pelvic pain. Obstet Gynecol 2003;101:594611.

3. Daniels JP, Khan KS. Chronic pelvic pain in women. BMJ 2010; 341:c4834.

4. Abrams P, Cardozo L, Fall M, Griffiths D, Rosier P, Ulmsten U, et al. Am J Obstet Gynecol 2002;187:116-26.

5. Carey ET, Till SR, As-Sanie S. Pharmacological management of chronic pelvic pain in women. Drugs 2017;77:285-301.

6. Gebhart GF, Bielefeldt K. Physiology of visceral pain. Compr Physiol 2016;6:1609-33.

7. McAllister SL, McGinty KA, Resuehr D, Berkley KJ. Endometriosis-induced vaginal hyperalgesia in the rat: role of the ectopic growths and their innervation. Pain 2009;147(1-3):255-64.

8. Giamberardino MA, Berkley KJ, Affaitati G, Lerza R, Centurione L, Lapenna D, et al. Influence of endometriosis on pain behaviors and muscle hyperalgesia induced by a ureteral calculosis in female rats. Pain 2002;95:247-57.

9. Morrison TC, Dmitrieva N, Winnard KP, Berkley KJ. Opposing viscerovisceral effects of surgically induced endometriosis and a control abdominal surgery on the rat bladder. Fertil Steril 2006;86(4 Suppl):1067-73.

10. Stratton P, Berkley KJ. Chronic pelvic pain and endometriosis: translational evidence of the relationship and implications. Hum Reprod Update 2011;17:327-46.

11. Hansen KE, Kesmodel US, Baldursson EB, Kold M, Forman A. Visceral syndrome in endometriosis patients. Eur J Obstet Gynecol Reprod Biol 2014;179:198-203.

12. Fariello JY, Whitmore K. Sacral neuromodulation stimulation for IC/PBS, chronic pelvic pain, and sexual dysfunction. Int Urogynecol J 2010;21:1553-8.

13. Khoder W, Hale D. Pudendal neuralgia. Obstet Gynecol Clin North Am 2014;41:443-52.

14. Possover M. Intractable neural pelvic pain. In: Gomel V, Brill AI, editors. Reconstructive and reproductive surgery in gynecology. London: Informa Health Care; 2010. p. 200-10.

15. Possover M, Forman A, Rabischong B, Lemos N, Chiantera V. Neuropelveology: new groundbreaking discipline in medicine. J Minim Invasive Gynecol 2015;22:1140-1.

16. McCorry LK. Physiology of the autonomic nervous system. Am J Pharm Edu 2007;71:78.
17. Markos AR, Dinsmore W. Persistent genital arousal and restless genitalia: sexual dysfunction or subtype of vulvodynia? Int J STD AIDS 2013;24:852-8.

18. Possover M, Forman A. Pelvic neuralgias by neuro-vascular entrapment: anatomical findings in a series of 97 consecutive patients treated by laparoscopic nerve decompression. Pain Physician 2015; 18:E1139-43.

19. Zanatta A, Rosin MM, Machado RL, Cava L, Possover M. Laparoscopic dissection and anatomy of sacral nerve roots and pelvic splanchnic nerves. J Minim Invasive Gynecol 2014;21:982-3.

20. Possover M, Schneider T, Henle KP. Laparoscopic therapy for endometriosis and vascular entrapment of sacral plexus. Fertil Steril 2011;95:756-8.

21. Lemos N, Possover M. Laparoscopic approach to intrapelvic nerve entrapments. J Hip Preserv Surg 2015;2:92-8.

22. Possover M, Baekelandt J, Chiantera V. The laparoscopic implantation of neuroprothesis ( $\mathrm{LION}$ ) procedure to control intractable abdomino-pelvic neuralgia. Neuromodulation 2007;10:18-23.

23. Possover M. A novel implantation technique for pudendal nerve stimulation for treatment of overactive bladder and urgency incontinence. J Minim Invasive Gynecol 2014;21:888-92.

24. Chakravarthy K, Nava A, Christo PJ, Williams K. Review of recent advances in peripheral nerve stimulation (PNS). Curr Pain Headache Rep 2016;20:60.

25. Banakhar M, Hassouna M. Sacral neuromodulation for genitourinary problems. Prog Neurol Surg 2015;29:192-9.

26. Nordenstam J, Boller AM, Mellgren A. Sacral nerve stimulation in the treatment of bowel disorders. Prog Neurol Surg 2015;29:20012.

27. Possover M. The LION procedure to the pelvic nerves for recovery of locomotion in 18 spinal cord injured peoples - a case series. Surg Technol Int 2016;XXIX:19-25.

28. Possover M, Forman A. Recovery of supraspinal control of leg movement in a chronic complete flaccid paraplegic man after continuous low-frequency pelvic nerve stimulation and FES-assisted training. Spinal Cord Ser Cases 2017;3:16034.

29. Quaghebeur J, Wyndaele JJ. Chronic pelvic pain syndrome: role of a thorough clinical assessment. Scand J Urol 2015;49:81-9.

30. Quaghebeur J, Wyndaele JJ, De Wachter S. Pain areas and mechanosensitivity in patients with chronic pelvic pain syndrome: a controlled clinical investigation. Scand J Urol 2017;51:414-9. 